\title{
CAP-ONES: An Emergency Notification System for all
}

\author{
Alessio Malizia, Pablo Acuña, Teresa Onorati, Paloma Díaz, Ignacio Aedo \\ Departamento de Informática \\ Universidad Carlos III de Madrid, Spain \\ \{amalizia, pacuna, tonorati, pdp\}@inf.uc3m.es, aedo@ia.uc3m.es
}

\begin{abstract}
In this paper we present an ontology-based system for managing emergency alert notifications. Our purpose is to generate emergency alerts that are accessible to different kinds of people, paying special attention to more vulnerable collectives like impaired people. By adapting alerts to different devices and users we can allow Emergency Management Systems (EMS) to communicate with collectives like blind or deaf people whom otherwise will be unreachable by usual channels. Moreover, if we consider the constrains imposed by the nature of the emergency situations we can also improve the information transmission to cope with situational disabilities (e.g. smoke during a fire can cause low vision problems). We centered our system architecture on two characteristics: the first one is an ontology that codifies knowledge about accessibility, devices, disabilities, emergencies and media so the alert notification can be tailored according to different parameters; the second one is the use of an open standard like the CAP (Common Alerting Protocol) that enables our system to interoperate with other existing systems.
\end{abstract}

\section{Keywords}

Concepts and Models for Crisis Ontologies, Alerting systems, Emergency Response Information Systems, Accessibility, Design for all

\section{INTRODUCTION}

Communicating alerts in a useful and efficient way is vital to reduce the number of victims in emergency situations but this is a challenging goal considering the diversity of users and circumstances involved in an emergency. Most Emergency Management Systems (EMS) include Emergency Notification Systems (ENS) in form of separate systems or modules. They are used for notifying emergency alerts by means of different media, such as: sms, mms, e-mail or Internet when available. ENSs are intended to deliver information to as many devices as possible, in order to reach people using different kinds of hardware. However, to the best of our knowledge, all the existing ENS do not include or include only partially accessibility aspects adapting messages formats according to people's profiles and preferences, as surveyed in [1]. Moreover we found that the main media used for emergency notification is the Internet with mobile or traditional devices [1]. We should also take into account the emergency notifications not only depend on people's abilities and on the devices characteristics, but also on the kind of emergency, which could inhibit the device capabilities or change the user's characteristics. For instance, visual interfaces might be useless for blinds but also in situations like wildfires with scarce visibility. Therefore, ENS should transmit messages using the most appropriate media and format taking into account the capabilities and limitations of people and devices as wells as those imposed by the emergency situation. In this way, the ENS will support alerts for all as a specific kind of "Universal Design" or "Design for All” [2] as depicted in Figure 1.

Another important issue to consider in the context of EMS is interoperability. Large scale emergencies involve different organisms and agencies each of which has its own system and, therefore, emergency alerts should be communicated over different channels. The CAP (Common Alerting Protocol) 1.0 standard specification approved by the OASIS consortium [3] provides a conceptual framework to achieve this interoperability. OASIS ${ }^{1}$ is the Organization for the Advancement of Structured Information Standards, and CAP is an XML-based data format for interchanging warnings and emergencies between alerting technologies. CAP is focused on defining and exchanging the different kind of alerts and types of notifications but does not take into account the different abilities of the users

$1 \quad$ http://www.oasis-open.org/home/index.php

Proceedings of the $6^{\text {th }}$ International ISCRAM Conference - Gothenburg, Sweden, May 2009

J. Landgren and S. Jul, eds. 
and does not, explicitly, model the relationships among the technologies and the kinds of emergencies.

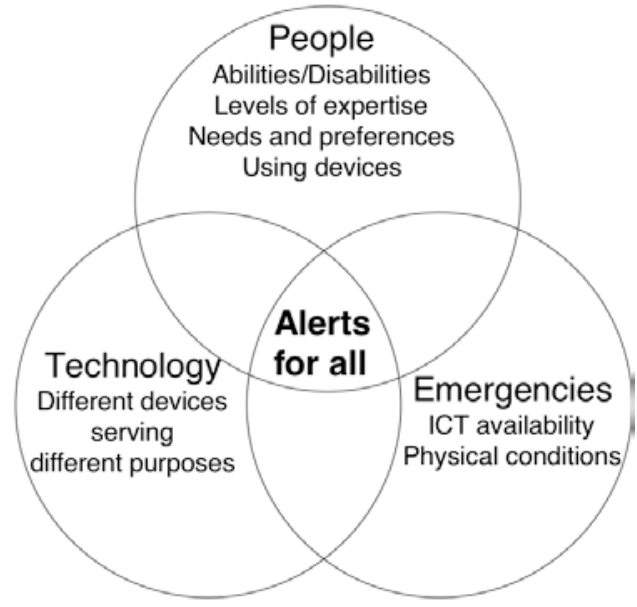

Figure 1. The emergency alerts information space.

In this context we have generated a structured knowledge base in the form of an ontology to link all the dimensions of the alert notifications information space (type of notification, users' abilities to react or understand the notifications, available technologies. We have built a prototype called CAP-ONES (Common Alerting Protocolbased Open Notification System) that, reasoning over the ontology's knowledge, can automatically generate the most adequate notification according to the user, the emergency and the device features. In this way, we provide automatic support for notifications for all without any intervention from the EMS operator. Moreover, since the prototype is triggered when CAP notifications are received it could be integrated with any existing EMS generating this standard alert format.

The remaining of the paper is structured as follows. Section 2 describes the proposed ontology centralizing and modeling the knowledge of the emergency alert information space. In the Section 3 we illustrate the prototype, its architecture and components. Next section shows the system evaluation by using ontology validation and use cases. We conclude with a section commenting the characteristics of the system and describing future works.

\section{THE SEMA4A ONTOLOGY}

SEMA4A (Simple Emergency Alerts fo[u]r All) is the ontology we have developed to gather and interrelate the knowledge underlying the emergency alerts space. It counts on three basic classes (EMEDIA, WAfA, and AccessOnto) that provide information related to the concepts and relations needed to model organization, structure and navigation of information items; accessibility guidelines, user's profiles and actions that users can perform; as well as information related to emergencies, notifications and devices. These main classes are linked through a number of relations defined amongst their subclasses. We give here a brief description of the main classes and concepts included in the SEMA4A ontology to give an overall idea of the specific knowledge codified in it. For a thorough description of the ontology see [1].

- EMEDIA (Emergency and MEDIA technologies) is the portion of the SEMA4A ontology that we have created to model concepts and relations about emergency and media technologies. We developed it trough a semiautomatic procedure with two phases: the first phase was performed consisted of extracting new concepts and relations concerning emergency and media technologies from WordNet [4]; the second one integrated new information within the existing ontology adding relations with the others classes of SEMA4A, WAFa and AccessOnto [5,6]. We applied this technique to develop and expand part of our ontology related to the emergencies and how they can affect technologies accessible to the users.

- The Web Authoring for Accessibility (WAfA) ontology represents concepts and relations necessary to automatically model the structural organization and navigation of web pages [5] to users' profiles. This ontology has been evaluated with real users, and contains information on how to model content for being accessible, and it is codified using the Web Ontology Language (OWL) ${ }^{2}$. We extended our ontology

2 http://www.w3.org/TR/owl-guide/

Proceedings of the $6^{\text {th }}$ International ISCRAM Conference - Gothenburg, Sweden, May 2009

J. Landgren and S. Jul, eds. 
including WAfA concepts, defining a class called WAfA that contains concepts and relations needed to model organization, structure and navigation of sites.

- AccessOnto in an existing ontology in form of an accessibility requirements repository from which it is possible to extract requirements using an accessibility knowledge base (AKB) built on user's characteristics [6]. It includes guidelines from Web Accessibility Initiative, Sun Micro Systems, IBM, Microsoft, and Apple guidelines. In our ontology we created a class called AccessOnto that contains information related to Web accessibility guidelines, users' profiles and actions that users can perform. We created this class translating information from XML (AccessOnto is codified in XML) to OWL.

The SEMA4A ontology has been developed using an editor ${ }^{3}$ from Stanford University called Protégé 3.2.1, and it is codified in OWL. It was also used an OWL DL ${ }^{4}$ reasoning tool from Mindswap laboratory at Maryland University called Pellet to verify consistency of the ontology classes.

\section{THE CAP-ONES SYSTEM}

Taking as basis the SEMA4A ontology, we developed a prototype for automatically creating and sending personalized emergency notifications using different media and devices. The main idea is to extract from the ontology the media and devices which better fit the abilities of the users and the information available from the emergency.

In a nutshell, the prototype gets two inputs: an emergency alert in a CAP XML format and a set of user profiles that include their abilities and available devices. Our system (Figure 2) receives both inputs and parses the information. The systems parses the CAP and Profiles files to extract all the relevant information and standardizes them into a fixed internal representation (based on XML). Using this information it performs queries on the SEMA4A ontology in order to obtain the potential ways to send the alert according to users' abilities and the kind of emergency. In fact, not only the profile counts but also the kind of emergency since there are emergencies like hurricanes that can affect the communication infrastructures and thus the type of alert that could be sent (as in the case of Katrina where sms were used since phone lines and Internet connections were down). The queries results give us a set of media and devices that can be used to adapt a notification for each profile. Next paragraphs describe all the component of the prototype.

\section{Emergency Alerts module}

The emergency information to be processed is extracted from the Common Alerting Protocol (CAP) message. A CAP message is formatted using XML and contains information about emergency alerts and notifications including: its purpose, source, status, description, instructions, etc., as well as a flexible set of additional information that can contain geographic data with different formats, resources (video, images and/or text files) and system-specific parameters associated with the emergency alert. We decided to use CAP standard in order to allow a future interoperability with additional systems able to issue emergency notifications, allowing our prototype to obtain such information and perform the corresponding operations to issue the notifications. The current version of the system allows the user to enter information via a web page (Figure 2A) or by importing a CAP file or directly providing an URL pointing at a CAP file. For instance, CAP messages could be received through different ways extending our prototype emergency input, allowing interoperability with other systems, e.g. a system producing CAP messages that can become our input to generate accessible notifications, like the EDIS (Emergency Digital Information Service), provided by California Office of Emergency Services ${ }^{5}$, that includes a CAP repository to deliver emergency information to the public and media.

\section{Profiles module}

The profiles file is the second input of the prototype, and contains information of users in order to relate each user's profile abilities with information available from the emergency alert (in CAP format). The profiles are created using a web interface (Figure 2B) that allows entering:

3 http://protege.stanford.edu/

4 http://pellet.owldl.com/

5 http://edis.oes.ca.gov/.

Proceedings of the $6^{\text {th }}$ International ISCRAM Conference - Gothenburg, Sweden, May 2009

J. Landgren and S. Jul, eds. 
- Personal and contact information: name, location data (address, country), mobile phone number, e-mail.

- Abilities: levels of user's abilities (low, medium or high) in 6 different categories including: Cognitive, Hearing, Coordination, Tactile Sensation, Visual and Colour. The 6 categories are codified in the ontology, as well as their properties and restrictions. We use positive abilities so that not only permanent disabilities (for example, being deaf) can be considered but also situational ones (for example not being able to hear due to the noise).

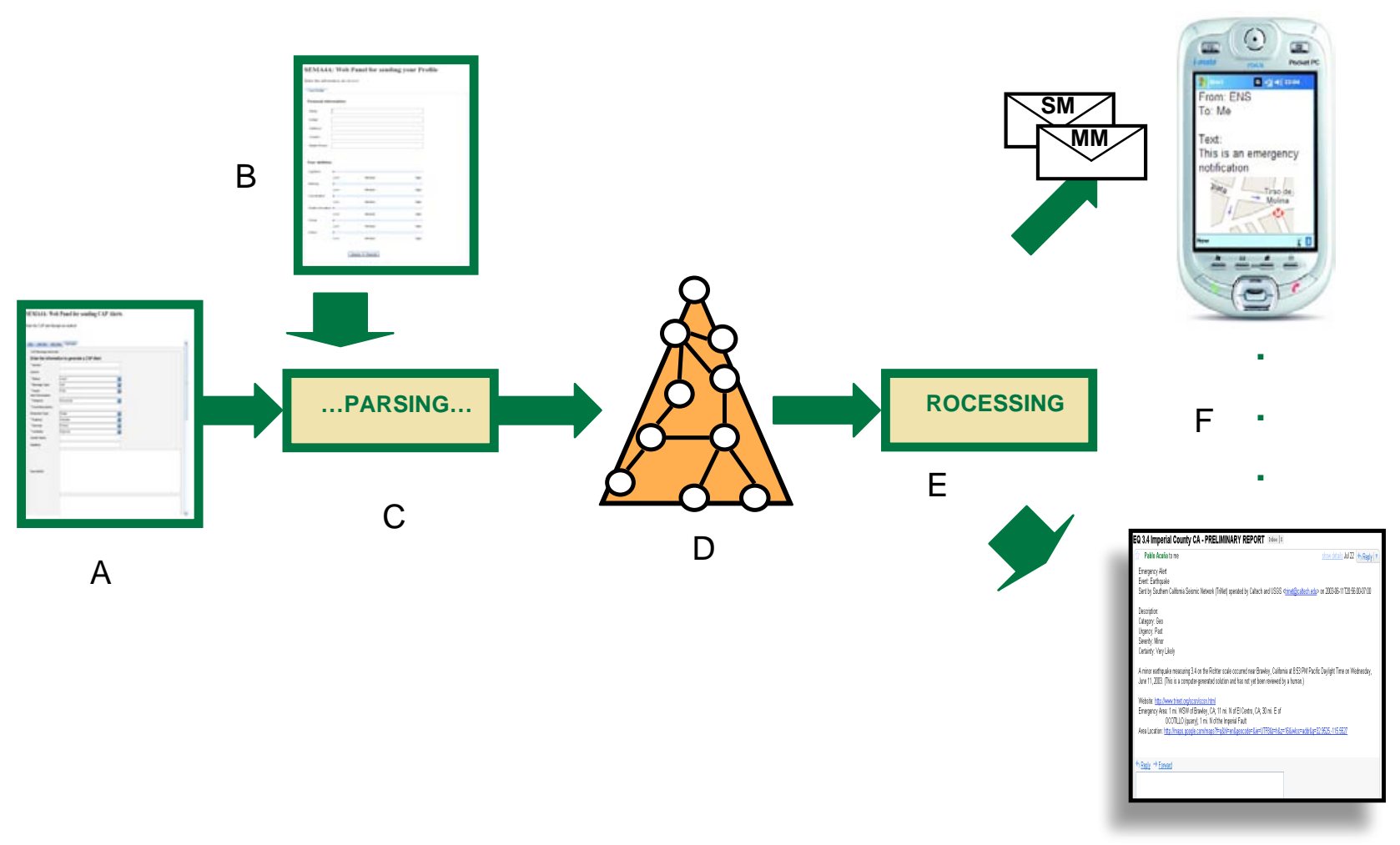

Figure 2. System architecture: (A) web page allowing entering CAP Alerts as input of the system, (B) profiles with specific information from the users, serving as input, (C) component that receives both inputs and parses the information related to the emergency and the users capabilities, (D) the system sends queries to the ontology using the information extracted from the inputs, (E) component that processes the data obtained from the ontology in order to adapt personalized notifications, (F) individual notifications issued to profiles obtained from input (B) using a specific media with a specific format according to the information available.

- Possible devices: depending on the user's level entered in the abilities section, a set of possible devices extracted from the relationships defined in the ontology, is presented, and the user must select all the possible combination of media that he or she can use to receive the emergency notifications, e.g. e-mail, sms, mms, etc.

Profiles could be entered in the system by the user itself or by assistants or relatives (in case he/she cannot do it). Profiles are stored as static xml files in the current version of the prototype, even if we plan to allow them to change dynamically according to different situations the user could encounter or devices he/she could be equipped with.

\section{Ontology Queries}

Once all the information from the emergency and profiles is retrieved from the CAP emergency alert and the user profiles entered in the system, a series of queries are executed on the ontology in order to get the media and devices that can be used in order to issue a personalized notification accessible to each user.

There are different implementations that allow creation of queries to Ontology. In our system we utilize SPARQL

Proceedings of the $6^{\text {th }}$ International ISCRAM Conference - Gothenburg, Sweden, May 2009

J. Landgren and S. Jul, eds. 
(Simple Protocol and RDF Query Language) ${ }^{6}$, a W3C Recommendation that allows querying information to a RDF (Resource Description Framework) document. Based on the ontology structure and the available information from the emergencies in a CAP alert and the profiles, the system performs the following queries on the SEMA4A ontology:

(1) Retrieve the kind of emergency as defined in the ontology that better fits the description of the alert included in the CAP file. The ontology includes definition of emergency classes and their properties, such as severity, urgency and certainty, included in CAP. SPARQL allows text operations to be performed on the class names and data used in the queries. This way, a class name can be compared to the description of the emergency using a regular expression. Every class name that matches using regular expressions is evaluated and a class name that best fits to the description of the emergency is used for next steps.

(2) Retrieve the media that may be used in of the kind of selected emergency. SEMA4A ontology defines a series of media and relates them with emergencies using a property (restriction) mayUse.

(3) Retrieve what can be communicated through the media obtained in step (2). SEMA4A includes relationships between media and different tools that can be used to communicate, e.g. e-mail, sms, etc., using RDF property can-communicate.

(4) For each profile, retrieve the media that the user is able to manage, depending on his/her profile information. SEMA4A defines relationships between impairments and media using the same property mentioned in step (2) (mayUse); and relationships between impairments and media tools using property mentioned in step (3) (can-communicate).

Let's consider a CAP emergency alert describing an earthquake in a specific location. First we need to obtain the media that may be used by that emergency, creating a SPARQL query relating the emergency class Earthquake using the OWL restriction mayUse (included in our ontology); the results of query (1) for the specific case of an Earthquake will be: $t v$, radio, mobile_phone, phone, internet, eye_tracking.

Successively, we need to obtain the devices related to the media that can be used to send the emergency notification, using the OWL restriction can-communicate included in SEMA4A; so the result of query (2) is: Video, Sound, multiple_languages, Figure, Text, mms, email, sms, vibration.

Each profile contains a set of media and devices that can be used according to users' abilities, as previously described in Profiles section. Let's consider a profile with deafness disability for this specific case. The query to obtain the mentioned set of media and devices is (3) and the results will be: mms, vibration, sms, Figure, Video, Text, text_enhancer, email.

Finally we compute the intersection between the set of media that the emergency may use and the media used by the profile (first and third result). Then, we compute the intersection between the set of devices that the emergency can communicate and the media used by the profile. The final set is the union of the previous ones that represents the kinds of media usable depending on user's abilities and emergency. The set in the presented case is made of: Figure, Text, mms, email, sms, vibration.

\section{Notifications}

Using the final result set obtained from the queries executed on SEMA4A ontology for a specific emergency and user profile, we have the media and the devices that can be used to issue a notification. In addition to this, we create a personalized message that suits the results and provides a particular notification to a user. This alert message is created by selecting the appropriate content from the CAP alert depending on users abilities and media selected for the specific emergency. Thus we adapt the multimedia content or geographic information contained in it to the different devices. We can, for example, send individual SMS with a formatted text including only relevant information due to the limited size, or MMS including multimedia resources included in the alert message. The prototype can be extended in order to support additional functionality.

\section{SYSTEM EVALUATION}

The prototype presented in this paper is a first step towards the development of a system for adapting alert notifications to different users. The current version deals with a specific set of disabilities (we are still implementing

6 http://www.w3.org/TR/rdf-sparql-query/

Proceedings of the $6^{\text {th }}$ International ISCRAM Conference - Gothenburg, Sweden, May 2009

J. Landgren and S. Jul, eds. 
adaptation for cognitive disabilities) and with a restricted set of devices (mobile phones, pdas and PC for now). For these reasons we opted for an analytic evaluation postponing the expertimental evalution with final users to a further level of implementation. Our analytic evaluation consists of two steps: the ontology validation, and the description and implementation of 2 use cases which are representative of the potential use of our system.

\section{Ontology Validation}

There exist many different methods and techniques to validate and evaluate ontologies; in an early work [1] we used an approach inspired by Spyns et al. [7] based on information extracted from the ontology and producing a quantitave evaluation by contrasting the information contained in the ontology with a corpus of documents concerning emergency and accessibility. Differently from [1] we have applied here a qualitative evaluation. Domain experts were asked to evaluate the value and usefulness of the concepts and facts extracted from the ontology. In fact, according to the HCOME Methodology [8] knowledge workers should participate actively in the ontology engineering processes. We selected two evaluators: one is an expert of accessibility who worked several years for R\&D projects; she is particularly expert on Infometrics (information measurement) applied to web accessibility. The second evaluator is an expert professional working for Spanish Civil Protection and developing documents, policies and recommendations on the emergency domain. We evaluated both the accessibility and emergency aspects of the knowledge contained in SEMA4A. Questions have been asked in form of a short evaluation questionnaire associated to the elements extracted from the ontology and for the respective domains. We generated two different sets of elements: one including only concepts and facts on emergency evaluated by the emergency expert, and another one with accessibility evaluated by the accessibility expert. The expert on accessibility evaluated, totally, 155 elements extracted from our ontology.

Results were:

- Coverage $91 \%$ (have all the lexons to be discovered actually been discovered?)

- Precision $84 \%$ (are the lexons making sense for the domain?)

- Accuracy $79 \%$ (are the lexons not too general but reflecting the important terms of the domain?).

The expert on emergency evaluated, in total, 265 elements extracted from our ontology.

Results were:

- Coverage $66 \%$

- Precision $65 \%$

- Accuracy $45 \%$

These results were mainly due to the fact that the emergency part of our ontology was automatically built by extracting relevant information from corpus of documents suggested by experts; while the accessibility part was built by integrating ontologies that were already verified and cleaned. Nevertheless, this is a first step towards integrating the knowledge on emergency and accessibility, and actively using it in a system for adapting emergency alert notifications to different types of users.

\section{Use Cases}

In this section we present examples explaining the behavior of the system according to types of emergency alerts and the users' profiles.

The system provides an interface to create profiles that allows entering personal and contact information, as well as defining the users' abilities within a scale (low, medium, high) in 6 categories: Cognitive, Hearing, Coordination, Tactile Sensation, Visual and Color. It is important to mention that these categories and its properties correspond to the ones defined in the SEMA4A ontology. Figure 3A shows the panel used in our system to enter users' abilities. 


\begin{tabular}{|c|c|c|c|c|}
\hline \multicolumn{4}{|l|}{ Your abilities: } & \multirow{3}{*}{$\begin{array}{l}\text { Your Profile } \\
\text { Your devices: }\end{array}$} \\
\hline Cognitive & 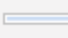 & & - & \\
\hline & Low & Medium & High & \\
\hline \multirow[t]{2}{*}{ Hearing } & . & & 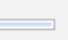 & $\square$ email \\
\hline & Low & Medium & High & $\square$ text_enhancer \\
\hline \multirow[t]{2}{*}{ Coordination } & & & - & $\square$ eye_tracking \\
\hline & Low & Medium & High & $\nabla$ Text \\
\hline \multirow[t]{2}{*}{ Tactile Sensation } & & & - & $\square$ Figure \\
\hline & Low & Medium & High & $\square$ Video \\
\hline \multirow[t]{2}{*}{ Visual } & & & - & $\checkmark$ vibration \\
\hline & Low & Medium & High & $\square$ sms \\
\hline \multirow[t]{3}{*}{ Colour } & 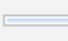 & & $\rightarrow$ & $\checkmark \mathrm{mms}$ \\
\hline & Low & Medium & High & Send \\
\hline & & (A) & & (B) \\
\hline
\end{tabular}

Successively it shows to the user the different media available according to the abilities she has defined in the first step, allowing selecting the media that she can utilize to receive notifications. The user can select many devices to define multiple ways to receive notifications. Figure $3 \mathrm{~B}$ shows the options for the profile in Figure $3 \mathrm{~A}$. This information is associated to the profile defined for the user.

Let's imagine having two different profiles inserted in our system. One is low hearing (corresponding to the deafness class in our ontology since it is an extreme condition) as presented in Figure 2, and the other one is low vision (low visual ability in the interface), that corresponds to blindness in our ontology (there are also other classes corresponding to intermediate situations like intermediate vision which corresponds to color blind or elderly people with problems of vision). So for low hearing ability (deafness) the selected media are: email, text, figure, vibration, mms, and sms. While for low vision ability (corresponding to blindness) we have: vibration, email, sound, speech, brailline.

Additionally, in this example, we use two emergency alerts in the form of a CAP XML message, an alert of an earthquake; and a Homeland Security Advisory System Update. The alerts are the following:

1. A CAP alert indicating an earthquake that occurred in California. The alert includes an area where the situation took place, including a circle definition with coordinates for latitude and longitude.

2. A CAP Alert indicating an update issued by The Department of Homeland Security where the threat level is elevated to ORANGE/HIGH. The alert contains an image as auxiliary resource, a textual description and a URL (Uniform Resource Locator) of its location.

Our system response to the scenarios described above is the following:

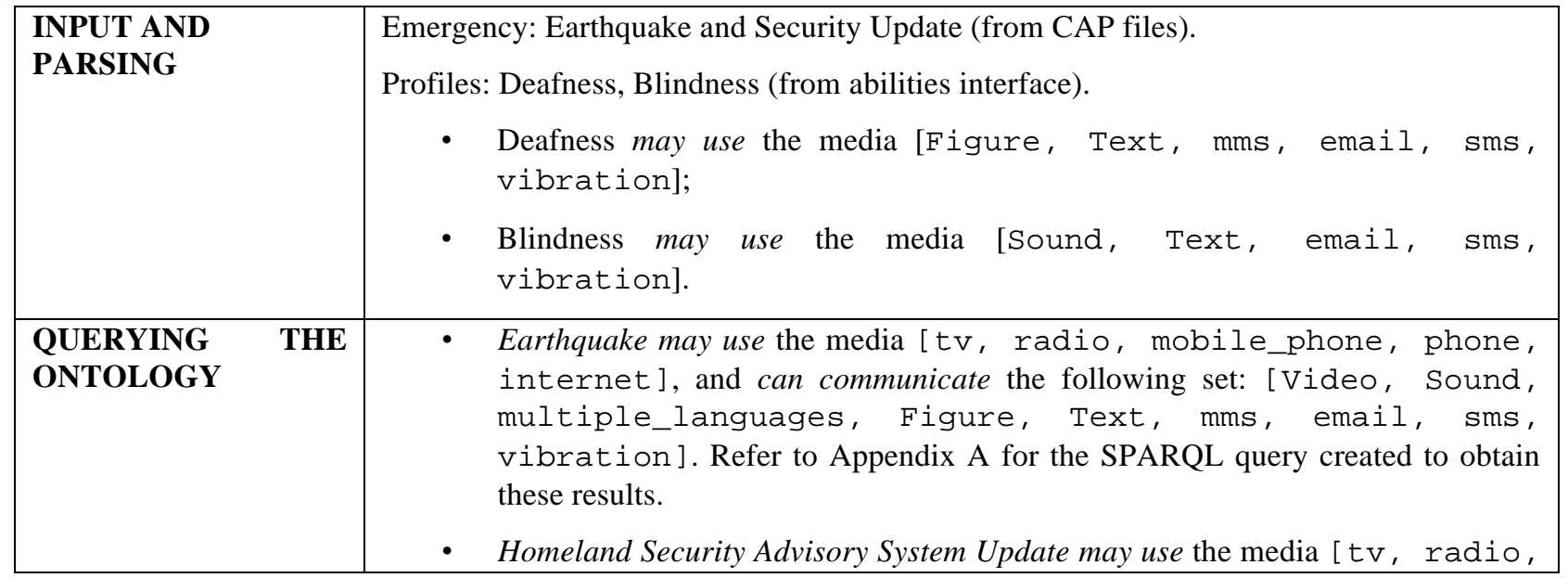

Proceedings of the $6^{\text {th }}$ International ISCRAM Conference - Gothenburg, Sweden, May 2009

J. Landgren and S. Jul, eds. 


\begin{tabular}{|c|c|}
\hline & $\begin{array}{l}\text { mobile_phone, phone, internet], and can communicate the } \\
\text { following set: [Video, Sound, multiple_languages, Figure, } \\
\text { Text, mms, email, sms, vibration]. }\end{array}$ \\
\hline PROCESSING & $\begin{array}{l}\text { Making an intersection with both sets (among profiles and emergencies), we can observe } \\
\text { that e-mail, mms and sms are feasible to send alert notifications to the deafness } \\
\text { profile. The profile Blindness can use only e-mail and sms to receive the notifications } \\
\text { (since a text can be processed by a screen reader software for blind people). }\end{array}$ \\
\hline \multirow[t]{2}{*}{$\begin{array}{l}\text { ALERT } \\
\text { PERSONALIZATION }\end{array}$} & $\begin{array}{l}\text { E-MAIL: since the earthquake emergency alert contained a geographical location of the } \\
\text { event, Google Maps }{ }^{7} \text { can be used to send additional information in the notification. } \\
\text { However, previous validation with the ontology must be performed. The emergency, } \\
\text { according to the ontology, can use Figure(s) in the notification; Deafness Profile may use } \\
\text { Figures as well, but Blindness profile cannot. According to this information, two different } \\
\text { e-mails are prepared and sent. The first e-mail (Figure 4) includes a link to Google Maps } \\
\text { in order to display the user with the location of the emergency, on the contrary, the } \\
\text { second mail (Figure 5) only includes a textual description of the area, in order to be read } \\
\text { by a specific device equipped with a screen reader or Braille technology. }\end{array}$ \\
\hline & $\begin{array}{l}\text { MMS/SMS: since the security update emergency alert contains an image to better } \\
\text { describe the situation, such image can be send within the notification. However, previous } \\
\text { validation with the ontology must be performed. The emergency, according to the } \\
\text { ontology, can use Figure(s) in the notification; Deafness Profile may use Figures as well, } \\
\text { but Blindness profile cannot. So to Blindness profile an SMS only message is sent. } \\
\text { According with this information, for example, two different sms and mms are prepared } \\
\text { and sent. The first mms (Figure 6) contains the image attached to the sent message, while } \\
\text { in the second, the sms (Figure 7) only includes the textual description of the image }\end{array}$ \\
\hline
\end{tabular}

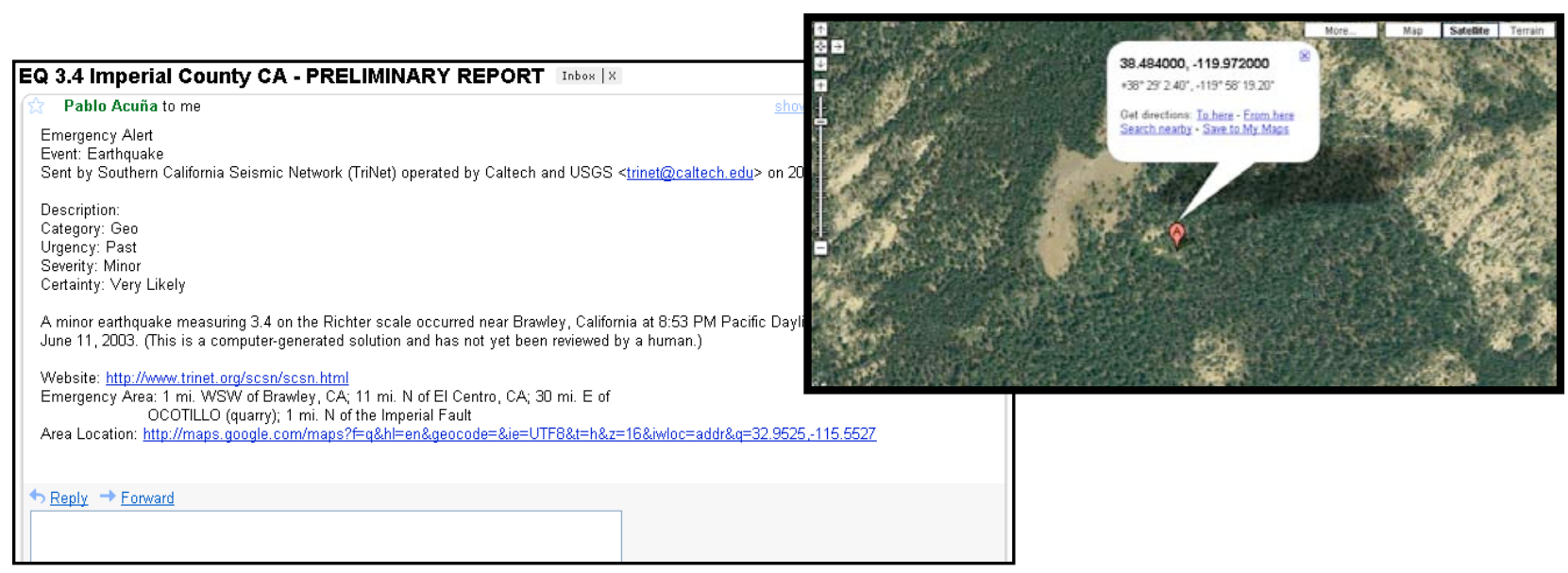

Figure 4: Alert notification through e-mail. In this particular case, considering the user's abilities a link to a map indicating the emergency place has been sent.

7 http://maps.google.com

Proceedings of the $6^{\text {th }}$ International ISCRAM Conference - Gothenburg, Sweden, May 2009

J. Landgren and S. Jul, eds. 


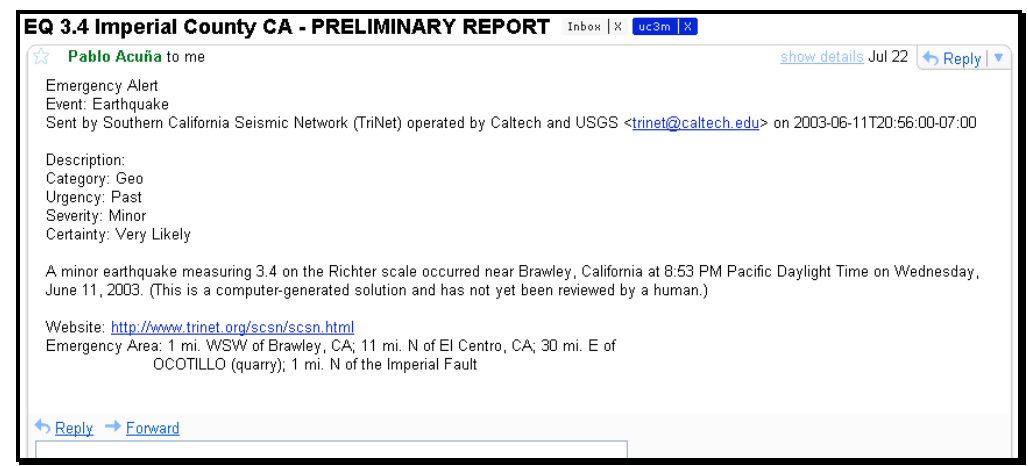

Figure 5: Alert notification through e-mail. Considering the user's abilities (blindness), in this case only a textual description of the emergency has been sent; this can be read by screen reader software.

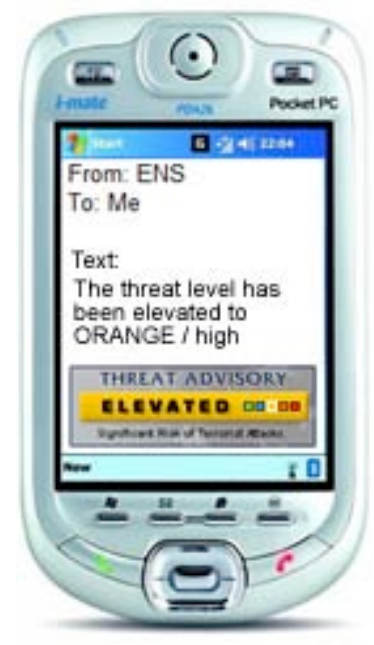

Figure 6: mms picture

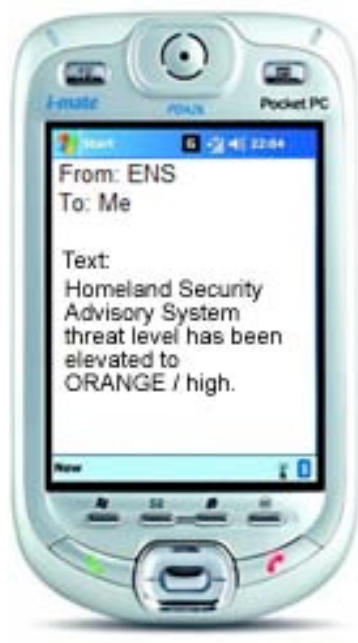

Figure 7: sms picture

\section{CONCLUSIONS AND FUTURE WORKS}

In this work we presented CAP-ONES a prototype for adapting alert notifications to different kinds of users depending on their abilities, the kind of emergency and the devices they can access. We presented the architecture of our system that supports the CAP standard for alert notifications which is important also to allow interoperability with existing systems. Furthermore, we described the shared knowledge in form of the SEMA4A ontology that proved to be valid in a precedent experiment and that we validated here contrasting the information included in it with experts' judgments. We also tested our systems with some use cases in order to show its value and flexibility in adapting alerts to different collectives of users with different abilities; we also managed different kinds of emergency and devices that they could access within such emergency scenarios. This is a first step toward a system that could automatically adapt alert notifications and interoperate with other systems but we think this is an important issue since accessibility in this particular case could mean help people in dangerous situations and reach them with the right information at the right time. In fact, we might consider that we are in some sense disabled when an emergency occurs: the smoke during a fire could reduce visibility or panic could interfere with our cognitive abilities; and thus adapting alerts could be of some help to a wide audience. Concluding we plan to research on ontology and system interoperability to let our system interoperate with other existing ones and to study new types of devices that could be useful in emergency situations and to adapt alert messages to these new kinds of devices. 


\section{ACKNOWLEDGEMENTS}

This work has been partly funded by the UIA4SIGE project (Ministry of Science and Innovation TSI2007-60388).

\section{REFERENCES}

1. Malizia, A., Astorga, F., Onorati, T., Diaz, P., Aedo I., (2008), Emergency Alerts for All: an Ontology based Approach to Improve Accessibility in Emergency Alerting Systems. ISCRAM $5^{\text {th }}$ International Conference Information Systems for Crisis Response and Management, 1:197-207, 2008.

2. Dix, A., Finley, J., Abowd, G., and Beale, R. 2003 Human-Computer Interaction (3rd Ed.). Prentice-Hall, Inc.

3. Steel, J., Iannella, R., Lam, H.P. Using Ontologies for Decision Support in Resource Messaging 5th International Conference on Information Systems for Crisis Response and Management, 5-7 May 2008, Washington DC, USA.

4. Yesilada Y. 2005. Annotation and transformation of Web pages to improve mobility for visually impaired users. PhD Thesis. School of Computer Science at the University of Manchester, UK.

5. Masuwa-Morgan, K. R., and Burrell, P. 2004. Justification of the need for an ontology for accessibility requirements (Theoretic framework). Interacting with Computers. Volume 16, Issue 3, June 2004, Pages 523555.

6. Miller, G., A., Beckwith, R., Felbaum, C., Gross, D., and Miller, K., (1990), "Introduction to WordNet : An Online Lexical Database", International Journal of Lexicography, Vol. 3, No. 4, 1990, 235 - 244.

7. Spyns, P., Meersman, R. and Jarrar, M., (2002), Data modelling versus ontology engineering. SIGMOD Record Special Issue, 31 (4): 12 - 17, 2002.

8. Kotis, K., Vouros, A., (2006), "Human-centered ontology engineering: The HCOME methodology", Knowledge and Information Systems 10 (2006) 109-131.

\section{APPENDIX A}

SPARQL Query to obtain the devices and media for a specific Emergency class (earthquake).

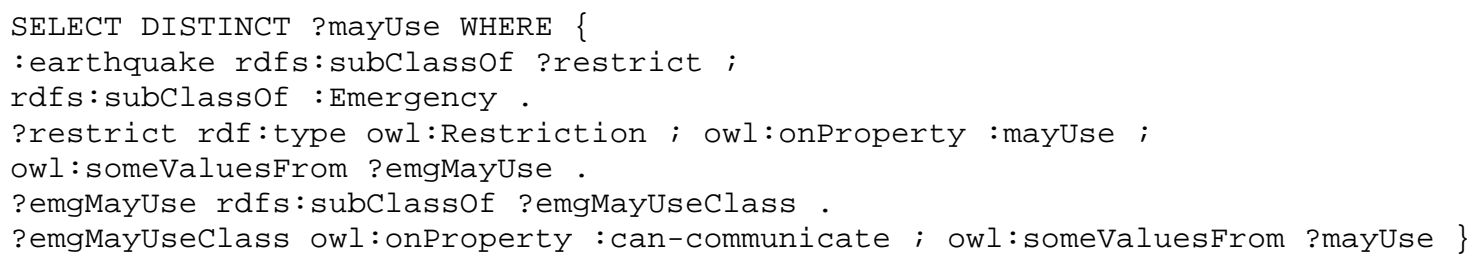

This query takes the earthquake class, a subclass of Emergency, and retrieves the object values from property mayUse, which gets the media that can be used for this emergency; from these media, the query retrieves the object values from property can-communicate in order to obtain the tools that can be used to send the notification.

Proceedings of the $6^{\text {th }}$ International ISCRAM Conference - Gothenburg, Sweden, May 2009

J. Landgren and S. Jul, eds. 